\title{
Interference effects in MSSM Higgs searches at the LHC
}

\section{Elina Fuchs*}

Weizmann Institute of Science, 760001 Rehovot, Israel

E-mail: elina.fuchs@weizmann.ac.il

\section{Georg Weiglein}

Deutsches Elektronen-Synchrotron (DESY), Notkestr. 85, 22607 Hamburg, Germany

E-mail: georg.weiglein@desy.de

\begin{abstract}
Complex parameters in the MSSM lead to mixing and interference between the two heavier neutral CP-even and CP-odd Higgs bosons. These effects can become very large in the case of almost degenerate states. In a CP-violating benchmark scenario, we investigate phenomenological implications of such interferences in view of the LHC searches for heavy Higgs bosons decaying to a pair of $\tau$-leptons and produced in gluon fusion and in association with $b$-quarks. Strongly destructive effects leave parameter regions unconstrained that would be regarded as excluded if no interference terms were taken into account.
\end{abstract}

38th International Conference on High Energy Physics 3-10 August 2016

Chicago, USA

${ }^{*}$ Speaker. 


\section{Introduction}

Searches for additional Higgs bosons have so far been interpreted within models beyond the Standard Model (BSM) by assuming the on-shell production of an unstable scalar and its subsequent decay. For quasi mass-degenerate particles that can appear as intermediate states between a given initial and final state, however, such a single-resonance approach or the incoherent sum of two resonance contributions does not necessarily hold. If the mass difference is smaller than the sum of their total width, the two resonances overlap. This can lead to a potentially large interference term, which is neglected in the standard narrow-width approximation (NWA), but can be taken into account in the full calculation or in a generalised NWA [1].

An important example for such a case are the two heavier neutral Higgs bosons of the Minimal Supersymmetric Standard Model (MSSM) which are almost degenerate in the decoupling limit. In the presence of complex MSSM parameters, they mix in a CP-violating way and interfere with each other. For heavy Higgs production in gluon fusion and in association with $b \bar{b}$ and the decay into a pair of $\tau$-leptons, we study the impact of such mixing and interference effects on the exclusion bounds within the MSSM with complex parameters (see also [2, 3]).

\section{MSSM Higgs sector with complex parameters}

Among the three neutral MSSM Higgs bosons, there are the $C P$-even states $h$ and $H$ as well as the $C P$-odd $A$. The charged Higgs bosons are denoted by $H^{ \pm}$. While the Higgs sector is $C P$ conserving at lowest order, non-vanishing imaginary parts of MSSM parameters enter the Higgs sector via loop corrections, leading to $C P$-violating mixing of the neutral $C P$-eigenstates $i=h, H, A$ into the mass eigenstates $h_{1}, h_{2}, h_{3}$ with masses $M_{h_{a}}$ and total widths $\Gamma_{h_{a}}, a=1,2,3$. The corresponding mixing factors are given by the on-shell wave function normalisation factors $\hat{\mathbf{Z}}_{a i}$ evaluated at the complex poles $\mathscr{M}_{h_{a}}^{2}=M_{h_{a}}^{2}-i M_{h_{a}} \Gamma_{h_{a}}$ which can be obtained from FeynHiggs [10, 11, 12, 13, 14]. We adopt the renormalisation scheme of Ref. [15].

The phases of MSSM parameters such as the trilinear couplings $A_{f}$ of the sfermions $\tilde{f}$ and the mass parameters of gauginos, $M_{1}, M_{3}$, and higgsinos, $\mu$, are in particular bounded by constraints from electric dipole moments (see e.g. Refs. [16, 17, 18]). However, constraints on the phases $\phi_{A_{f_{3}}}$ of the trilinear couplings of the third generation are weaker than for the first two generations. In addition, $\phi_{A_{t}}$ has the largest impact on the Higgs sector. Therefore we focus on the consequences of this phase for the mixing and interference effects and set $\phi_{A_{t}}=\phi_{A_{b}}=\phi_{A_{\tau}}$ in this work.

\section{3. $C P$-violating interference of MSSM Higgs bosons}

While for low and medium values of $\tan \beta$ the production of neutral MSSM Higgs bosons at the LHC is dominated by gluon fusion, $g g \rightarrow h_{a}(a=1,2,3)$, at high $\tan \beta$ the associated production with $b \bar{b}$ dominates. Regarding the decay, the $\tau^{+} \tau^{-}$- channel provides the strongest constraints [19, $20,21,22]$ for intermediate and large $\tan \beta$. For interference effects at low $\tan \beta$ in the $t \bar{t}$ final state, see $[24,25]$. We calculate the partonic cross sections of the following two $2 \rightarrow 2$ processes without NWA,

$$
b \bar{b} \rightarrow h_{a} \rightarrow \tau^{+} \tau^{-}
$$




$$
g g \rightarrow h_{a} \rightarrow \tau^{+} \tau^{-} .
$$

We compute process (3.1), which is part of the $b \bar{b} h_{a}$-associated production, at tree-level, and process (3.2) with one-loop diagrams of the first vertex. Besides, we take propagator-type corrections into account by using Higgs masses, total widths and $\hat{\mathbf{Z}}$-factors from FeynHiggs at 2-loop order. The Higgs resonances are parametrised as Breit-Wigner propagators and the mixing is accounted for by $\hat{\mathbf{Z}}$-factors, which yields a viable approximation of the full propagators [2, 4]. The cross section of the complete process contains the coherent sum of the amplitudes with $h_{1}, h_{2}, h_{3}$-exchange in the $s$-channel, i. e. $\sigma_{\mathrm{coh}}=\sigma\left(\left|h_{1}+h_{2}+h_{3}\right|^{2}\right)$, including the interference term, which is neglected in the incoherent sum, $\sigma_{\text {incoh }}=\sigma\left(\left|h_{1}\right|^{2}+\left|h_{2}\right|^{2}+\left|h_{3}\right|^{2}\right)$, i.e. $\sigma_{\text {int }}=\sigma_{\text {coh }}-\sigma_{\text {incoh }}$, and we define

$$
\eta=\frac{\sigma_{\text {int }}\left(\phi_{A_{t}}\right)}{\sigma_{\text {incoh }}\left(\phi_{A_{t}}\right)} .
$$

In the case of $\phi_{A_{t}}=0$, only $h$ and $H$ can interfere whereas a non-vanishing value of $\phi_{A_{t}}$ induces a $3 \times$ 3 mixing and the interference between all of the neutral Higgs bosons $h_{1}, h_{2}, h_{3}$. For the numerical evaluation, we define a $C P$-violating benchmark scenario as a modification of the standard $M_{h}^{\bmod +}$ scenario [5] by setting $\mu=1000 \mathrm{GeV}$ (as also proposed in [5]) and introducing the complex phase $\phi_{A_{t}}=\frac{\pi}{4}$. In this $C P$-violating scenario, Fig. 3 shows the relative interference effect $\eta$ in the $M_{H^{ \pm}}-$ $\tan \beta$ plane, Fig. 1(a) for the $b \bar{b}$-initiated process (3.1), and Fig. 1(b) for gluon fusion (3.2), both with $\tau^{+} \tau^{-}$in the final state. Due to the approximate degeneracy of $M_{h_{2}}$ and $M_{h_{3}}$ in the decoupling limit and the significant mixing of $H, A$ into $h_{2}, h_{3}$ in sizeable parts of the parameter space, the interference term becomes very large in both processes. Around $M_{H^{ \pm}}=480 \mathrm{GeV}, \tan \beta=29$, the relative interference contribution ranges down to $\eta=-97 \%$, surrounded by a considerable, destructive interference "valley".

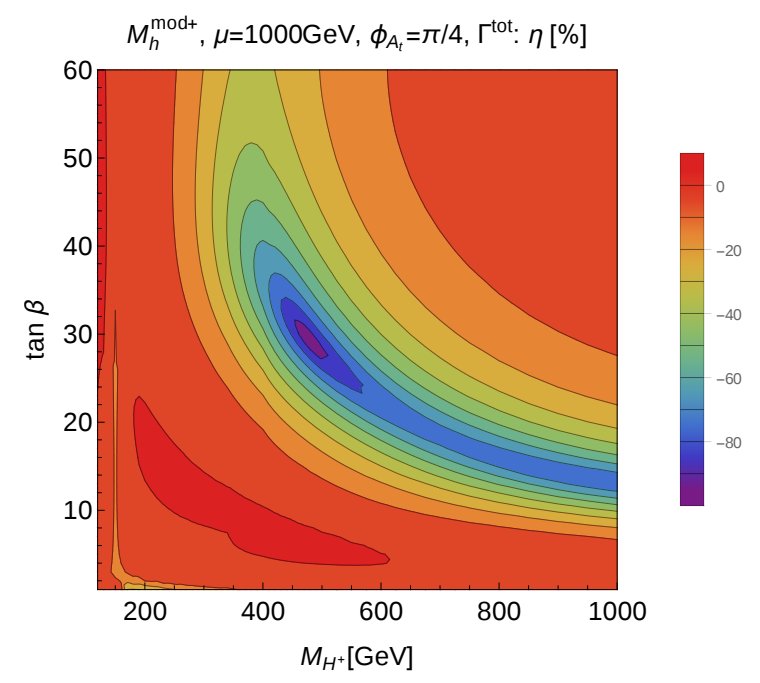

(a) $b \bar{b} \rightarrow h_{a} \rightarrow \tau^{+} \tau^{-}$.

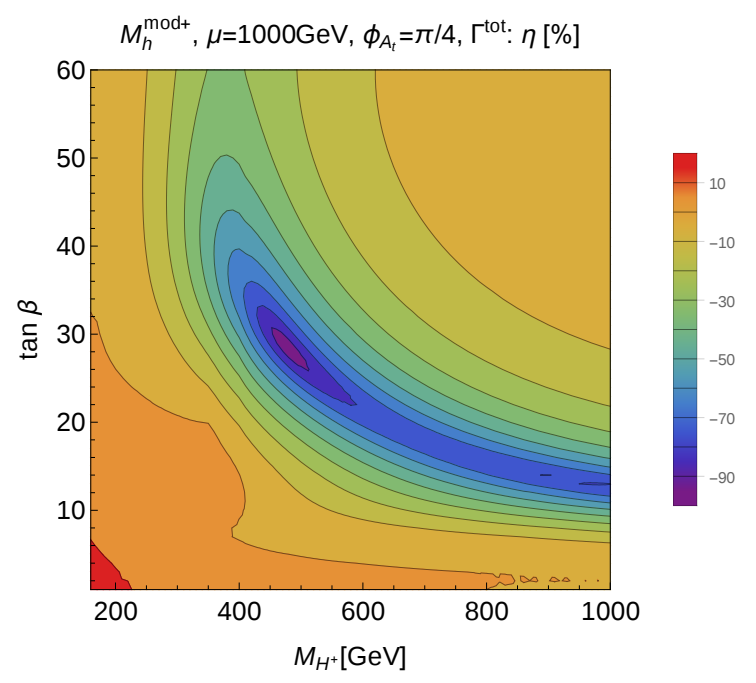

(b) $g g \rightarrow h_{a} \rightarrow \tau^{+} \tau^{-}$.

Figure 1: Relative interference contribution $\eta[\%]$ of the Higgs bosons $h_{1}, h_{2}, h_{3}$ decaying to $\tau^{+} \tau^{-}$in the complex $M_{h}^{\bmod +}$-scenario with $\mu=1000 \mathrm{GeV}$ and $\phi_{A_{t}}=\frac{\pi}{4}$. (a) $b \bar{b}$ initial state, (b) $g g$ initial state (note the different scale of the colour code). 


\section{Impact of interference terms on exclusion limits}

The significant, destructive interference terms suppress the expected cross sections and need to be included in a consistent evaluation of exclusion bounds. In our approach, we combine the existing precise predictions for separate Higgs production and decay (here from FeynHiggs -2 . 10.2 $[10,11,12,13,14])$ with the interference contribution. Our calculation of the interference effect includes propagator corrections, but no vertex and real corrections, which factorise and are already accounted for in the production cross sections and branching ratios.

In order to take the interference effect into account in the comparison of the model prediction and LHC data within HiggsBounds [6, 7, 8, 9], the overall interference term per process is split into the three combinations of two interfering Higgs bosons, respectively, $\sigma_{\text {int }}=\sigma_{\text {int }_{12}}+\sigma_{\text {int }_{13}}+$ $\sigma_{\text {int }_{23}}$. The individual cross sections $\sigma_{h_{a}}$ of each of the Higgs bosons $h_{a}$ are modified by the relative interference contribution

$$
\eta_{a}^{P}=\frac{\sigma_{\mathrm{int}}^{P}}{\sigma_{h_{a}}^{P}+\sigma_{h_{b}}^{P}}+\frac{\sigma_{\mathrm{int}}^{P}}{\sigma_{h_{a}}^{P}+\sigma_{h_{c}}^{P}},
$$

where $a, b, c=1,2,3$, and $P=g g, b \bar{b}$ denotes the production mode. The relative interference contributions $\eta_{a}^{P}$ are used to rescale the ratio of the production cross sections $P \rightarrow h_{a}$ in the MSSM with respect to the SM as input for HiggsBounds in the following way,

$$
\frac{\sigma^{\mathrm{MSSM}}\left(P \rightarrow h_{a}\right)}{\sigma^{\mathrm{SM}}\left(P \rightarrow h_{a}\right)} \longrightarrow \frac{\sigma^{\mathrm{MSSM}}\left(P \rightarrow h_{a}\right)}{\sigma^{\mathrm{SM}}\left(P \rightarrow h_{a}\right)} \cdot\left(1+\eta_{a}^{P}\right),
$$

leaving branching ratios $\mathrm{BR}\left(h_{a} \rightarrow \tau^{+} \tau^{-}\right)$unchanged.

Fig. 2 shows the resulting exclusion bounds obtained with HiggsBounds -4.2 . 0 (including Higgs search results up to run 1 of the LHC) with MSSM input from FeynHiggs, in the complex $M_{h}^{\text {mod+ }}$-scenario with $\mu=1000 \mathrm{GeV}$ and $\phi_{A_{t}}=\frac{\pi}{4}$. The augmented value of $\mu$ with respect to the default value of $200 \mathrm{GeV}$ leads to an increased sensitivity of the $h_{a} \rightarrow \tau \tau$ channel and enhances the $C P$-violating effects caused by the product $\mu A_{t}$ where $A_{t}$ has an imaginary part. The blue area represents the parameter space that seems to be excluded at the $95 \%$ confidence level (CL) in this scenario if the interference term is neglected by employing the standard NWA despite the presence of the complex phase $\phi_{A_{t}}$. This exclusion limit based on the CP-violating $3 \times 3$ mixing of $h_{1}, h_{2}, h_{3}$ is stronger than in the case of real parameters (with only CP-conserving $2 \times 2$ mixing) because the large off-diagonal elements in the full $\hat{\mathbf{Z}}$-mixing matrix enhance each individual production cross section $\sigma\left(P \rightarrow h_{a}\right)$ for $a=2,3$ in the region where $h_{2}$ and $h_{3}$ are quasi degenerate. The grey line corresponds to the case where the interference term is only included in the $g g$-initiated process whereas the inclusion of the interference term in the $b \bar{b}$ process gives rise to the black line. In contrast, the interference term is taken into account in both production processes in the 95\% CL exclusion contour displayed in red. The direct comparison shows that a substantial area between $M_{H^{ \pm}} \simeq 450 \mathrm{GeV}$ and $700 \mathrm{GeV}$ and roughly from $\tan \beta \simeq 18$ to 32 cannot be excluded in this scenario with complex parameters due to the strongly destructive interference effect observed in Fig. 3 in the same parameter region. This discrepancy between the conventional approach of the incoherent sum of separate Higgs contributions and the approach of including the interference among different neutral Higgs bosons shows that the standard NWA is insufficient in such a $\mathrm{CP}$ violating scenario. Interference between $h$ and $H$ can also play a role in the MSSM with real 


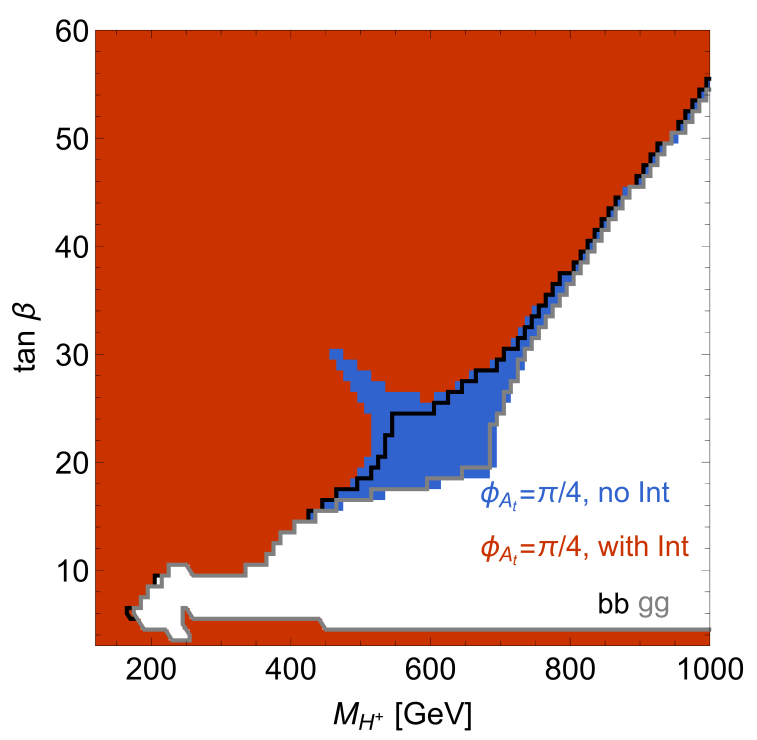

Figure 2: Exclusion bounds obtained with HiggsBounds in the complex $M_{h}^{\text {mod }+}$-scenario with $\mu=$ $1000 \mathrm{GeV}$ and $\phi_{A_{t}}=\pi / 4$ : without the interference term (blue), including the interference term in the $b \bar{b}$ and the $g g$ processes (red), including the interference only in $b \bar{b}$ (black line) or only in $g g$ (grey line).

parameters, but it becomes relevant only in a narrow parameter corner at low $M_{H^{ \pm}}$and very high $\tan \beta$, which lies in the deeply excluded region. However, the mass difference between $M_{h_{2}}$ and $M_{h_{3}}$ is smaller than $\Gamma_{h_{2}}+\Gamma_{h_{3}}$ in the major part of the parameter plane so that the $h_{2}-h_{3}$ interference term becomes important in the case of $C P$-violating mixing.

\section{Conclusions}

$C P$-violating mixing and interference of neutral Higgs bosons in the MSSM with complex parameters can have a significant impact on the interpretation of LHC searches for additional scalars. We have investigated a $C P$-violating benchmark scenario with a substantial mixing of $H$ and $A$ into the quasi-degenerate states $h_{2}$ and $h_{3}$. Both in $b \bar{b}$-associated Higgs production and in gluon fusion of $h_{1}, h_{2}, h_{3}$ decaying into a pair of $\tau$-leptons, a very large, destructive interference effect is found. The individual cross sections are enhanced by mixing effects whereas the combined expected cross section is substantially suppressed by the interference. As a consequence, a considerable parameter region, which would seem to be ruled out if the interference term were neglected, actually escapes exclusion by LHC Run 1 data. We will extend our analysis to the recent Run 2 results, additional complex phases and Higgs production cross sections from SusHiMi [23] that takes the full phase dependence into account.

\section{Acknowledgments}

We would like to thank Oscar Stål for his help with HiggsBounds and valuable suggestions, and Christian Veelken and Sven Heinemeyer for useful discussions. EF thanks the DESY theory group for hospitality. The work of E.F. was partially funded by the German National Academic 
Foundation and by DESY. The work of G.W. is supported in part by the Collaborative Research Centre SFB 676 of the DFG, "Particles, Strings and the Early Universe", and by the European Commission through the "HiggsTools" Initial Training Network PITN-GA-2012-316704.

\section{References}

[1] E. Fuchs, S. Thewes and G. Weiglein, Eur. Phys. J. C 75 (2015) 254.

[2] E. Fuchs, DESY-THESIS-2015-037.

[3] E. Fuchs and G. Weiglein, in preparation.

[4] E. Fuchs and G. Weiglein, arXiv:1610.06193 [hep-ph].

[5] M. Carena, S. Heinemeyer, O. Stål, C. E. M. Wagner and G. Weiglein, Eur. Phys. J. C 73 (2013) 9, 2552.

[6] P. Bechtle, O. Brein, S. Heinemeyer, G. Weiglein and K. E. Williams, Comput. Phys. Commun. 181 (2010) 138.

[7] P. Bechtle, O. Brein, S. Heinemeyer, G. Weiglein and K. E. Williams, Comput. Phys. Commun. 182 (2011) 2605.

[8] P. Bechtle, O. Brein, S. Heinemeyer, O. Stål, T. Stefaniak, G. Weiglein and K. Williams, PoS CHARGED 2012 (2012) 024.

[9] P. Bechtle, O. Brein, S. Heinemeyer, O. Stål, T. Stefaniak, G. Weiglein and K. E. Williams, Eur. Phys. J. C 74 (2014) 3, 2693.

[10] S. Heinemeyer, W. Hollik and G. Weiglein, "The Masses of the neutral CP - even Higgs bosons in the MSSM: Accurate analysis at the two loop level," Eur. Phys. J. C 9 (1999) 343. [hep-ph/9812472].

[11] S. Heinemeyer, W. Hollik and G. Weiglein, Comput. Phys. Commun. 124 (2000) 76. [hep-ph/9812320].

[12] G. Degrassi, S. Heinemeyer, W. Hollik, P. Slavich and G. Weiglein, Eur. Phys. J. C 28 (2003) 133.

[13] S. Heinemeyer, W. Hollik, H. Rzehak and G. Weiglein, Phys. Lett. B 652 (2007) 300.

[14] T. Hahn, S. Heinemeyer, W. Hollik, H. Rzehak and G. Weiglein, Phys. Rev. Lett. 112 (2014) 14, 141801.

[15] M. Frank, T. Hahn, S. Heinemeyer, W. Hollik, H. Rzehak and G. Weiglein, JHEP 0702 (2007) 047.

[16] V. D. Barger, T. Falk, T. Han, J. Jiang, T. Li and T. Plehn, Phys. Rev. D 64 (2001) 056007.

[17] Y. Li, S. Profumo and M. Ramsey-Musolf, JHEP 1008 (2010) 062.

[18] J. Engel, M. J. Ramsey-Musolf and U. van Kolck, Prog. Part. Nucl. Phys. 71 (2013) 21.

[19] CMS Collaboration [CMS Collaboration], CMS-PAS-HIG-14-029.

[20] G. Aad et al. [ATLAS Collaboration], JHEP 1411 (2014) 056.

[21] CMS Collaboration [CMS Collaboration], CMS-PAS-HIG-16-006.

[22] M. Aaboud et al. [ATLAS Collaboration], arXiv:1608.00890 [hep-ex].

[23] S. Liebler, S. Patel and G. Weiglein, in preparation.

[24] W. Bernreuther, P. Galler, C. Mellein, Z. G. Si and P. Uwer, Phys. Rev. D 93 (2016) no.3, 034032

[25] M. Carena and Z. Liu, arXiv:1608.07282 [hep-ph]. 